\title{
Clinical Features in Patients with Xq23 Microdeletion: A Case Report and Literature Review
}

\author{
(1) Lu Qin ${ }^{1}$, (1) Fei-Zhou Zhang1 ${ }^{1}$ (1) Jian-Hai Lv2 ${ }^{2}$ (1) Lan-Fang Tang2 \\ ${ }^{1}$ Children's Hospital of Zhejiang University School of Medicine, Department of Pulmonology, Zhejiang, China \\ 2Shangyu People's Hospital, Clinic of Pediatrics, Zhejiang, China
}

\begin{abstract}
What is already known on this topic?
Deletions in Xq22.3-q23 region may result in Alport syndrome, with intellectual disability, midface hypoplasia and elliptocytosis (AMME, MIM 300194), growth retardation, language delay, and alterations of bones, heart and eyes. Disorders affecting AMMECR1 can cause midface hypoplasia, elliptocytosis, language disorder including early speech or language delay, infantile hypotonia, hearing loss, nephrocalcinosis and submucous cleft palate, as well as short stature, cardiac and skeletal abnormalities. Another gene, CHRDL1, has been identified as the causative gene of X-linked megalocornea (MGC1; MIM 309300), which is associated with distinctive secondary changes in the posterior crocodile shagreen and corneal arcus juvenilis.
\end{abstract}

\section{What this study adds?}

We report a boy with Xq23 microdeletion, which involved both the AMMECR1 and CHRDL1 genes who presented with microsomia, midface hypoplasia, kidney dysplasia, growth retardation and some alterations of bones and heart. Furthermore, patients with Xq23 microdeletion or a deletion overlapping these genes partially, was reviewed to highlight the rare condition and analyze the genotypephenotype correlations.

\section{Abstract}

Xq22.3-q23 microdeletion is a rare genomic disorder. The purpose of this study was to emphasize the correlation between clinical phenotype and genotype of proximal deletion on chromosome Xq22.3-q23. A 5 years old boy had a $671 \mathrm{~KB}$ microdeletion on Xq23 by chromosomal microarray analysis, including AMMECR 1 and CHRDL1 genes. He presented with microsomia, midface hypoplasia, right kidney dysplasia and mildly motor retardation, which have not previously been reported in relation to Xq23 deletion. To the best of our knowledge, this is the first case with Xq23 microdeletion. A total of nine cases with microdeletion at Xq22.3-q23 affecting AMMECR 1 and two cases with CHRDL1 mutation were reviewed. This review showed that Xq23 microdeletion with microsomia, midface hypoplasia, kidney dysplasia, and mild motor retardation was rare. The previous literature showed two novel point mutations in AMMECR 1 and CHRDL1 with some phenotype difference from the presented case. Xq23 microdeletion should be considered for patients with microsomia, midface hypoplasia, kidney dysplasia and growth retardation.

Keywords: Xq23 microdeletion, midface hypoplasia, kidney dysplasia, growth retardation

\section{Introduction}

Xq22.3-q23 microdeletion is a rare genomic disorder, which encompasses the entire COL4A5 gene and its adjacent genes extending towards the telomere, including GUCY2F, NXT2, KCNE1L, ACSL4, TMEM164, MIR3978, AMMECR1, SNORD96B, RGAG1, TDGF3, CHRDL1, PAK3 and DCX (1). Deletions in this region may present with Alport syndrome, with intellectual disability, midface hypoplasia and elliptocytosis (AMME, MIM 300194), growth retardation, language delay, and alterations of bones, heart and eyes. The differences in phenotype are probably associated with the location and size of the deletion, which may include different genes. With array CGH technology, more patients with Xq microdeletion have been reported. To date, about 11 families and 21 patients with microdeletion at Xq22.3-q23 
have been reported. However, only two have been reported when the microdeletion has affected the AMMECR1 (MIM 300990) and Chordin-like 1 (CHRDL1 MIM 300350) genes, respectively. AMMECR 1 maps within the AMME complex interval (2). Disorder of this gene can cause midface hypoplasia, elliptocytosis, language disorder including early speech or language delay, infantile hypotonia, hearing loss, nephrocalcinosis and submucous cleft palate. In addition, AMMECR 1 is also associated with short stature, cardiac and skeletal abnormalities (2). CHRDL1 has been identified as the causative gene of X-linked megalocornea (MGC1; MIM 309300), which is associated with distinctive secondary changes in the posterior crocodile shagreen and corneal arcus juvenilis (3).

Herein, we report a patient with Xq23 microdeletion, which involved both the AMMECR1 and CHRDL1 genes who presented with microsomia, midface hypoplasia, kidney dysplasia, growth retardation and some alterations of bones and heart. Furthermore, patients with Xq23 microdeletion or a deletion overlapping these genes partially, was reviewed to highlight the rare condition and analyze the genotypephenotype correlations.

\section{Case Report}

A 5-year-old boy presented to our clinic for developmental delay with mildly motor retardation for 5 years. He weighed $3.3 \mathrm{~kg}$ at birth without any suspicion of asphyxia. At presentation he was Tanner stage $\mathrm{G}_{2} \mathrm{P}_{2}$ of nonconsanguineous, healthy parents. Antenatal screening implied right renal dysplasia and regular postpartum reexamination confirmed the diagnosis. Growth retardation with short stature were noted after birth. He was able to sit at the age of 9 months and walk at the age of 19 months. He could use simple words, such as "mum", at about 11 months but still stuttered. His right kidney was surgically removed at the aged of 3 years 8 months. During the operation, thin right ureter and multiple cysts in the left kidney were noted. Electrocardiogram and cardiac ultrasound examination showed type B pre-excitation syndrome and acleistocardia $(\Phi 0.27 \mathrm{~cm})$. Chest X-ray revealed no obvious abnormality of cardiopulmonary structures or diaphragm. Abdominal ultrasound scan did not show any abnormality of the liver, gallbladder or spleen. His father and mother were $163 \mathrm{~cm}$ and $153 \mathrm{~cm}$, respectively while his 8 year-old sister was also growing slowly [about $123 \mathrm{~cm},<-1$ standard deviation (SD)]. There was no family history of hereditary disease in the patient's family.

On physical examination, his height was $99.3 \mathrm{~cm}(<-2 \mathrm{SD})$. In addition, he had characteristic features, comprising flat facial profile, mildly epicanthic folds, downslanting palpebral fissures, flat nasal bridge, bulbous nose, smaller left cheek, and slightly asymmetrical nasolabial sulcus (Figure $1 \mathrm{~A}$ ). A flat lateral top of the skull, occipital protuberance, low-set ears, low hairline and short neck were also noted (Figure 1B). In addition, malpositioned teeth and bilateral clinodactyly of the fifth finger were also noted (Figure 1C). However, his intelligence was normal.

Laboratory examination of thyroid, liver and kidney function, gas chromatography/mass spectrometry for blood were regular or negative. X-ray scan showed seven ossification centers in the right-hand carpal bones. Chromosomal microarray analysis found a $671 \mathrm{~KB}$ microdeletion located at Xq23, which involved both the AMMECR1 and CHRDL1 genes (Figure 2). Unfortunately, the parents refused to perform further genetic testing.

We reviewed the literature in English (PubMed and OMIM database) and Chinese. A total of nine cases with
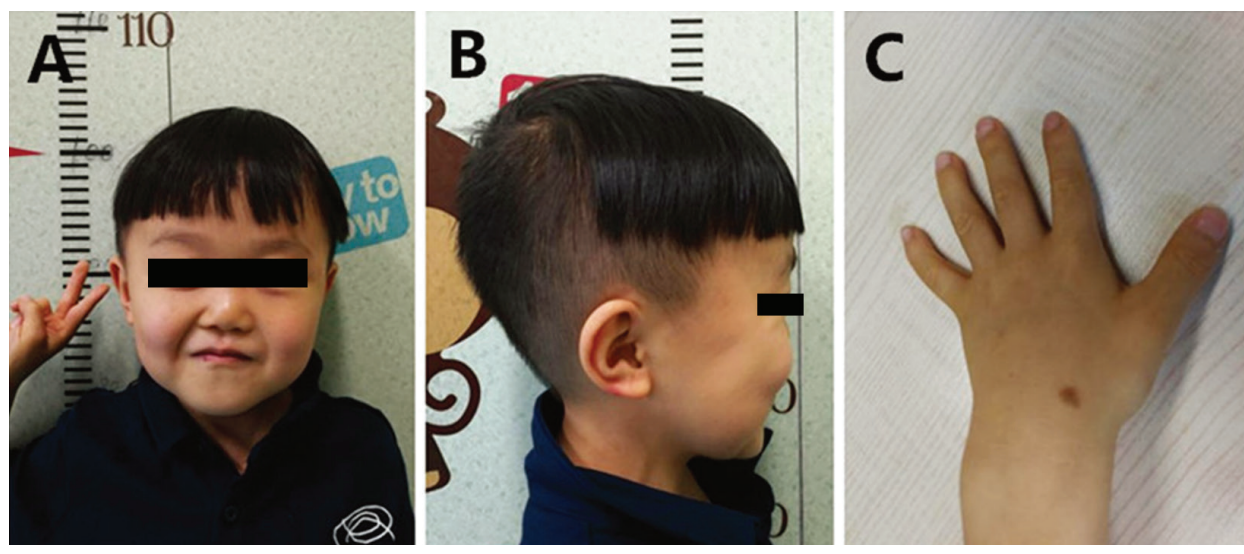

Figure 1. Clinical photographs of the current case. (A) Flat facial profile, mildly epicanthic folds, downslanting palpebral fissures, flat nasal bridge, bulbous nose, and short neck. The left cheek is smaller than the right cheek, and there is a slightly asymmetry of the nasolabial sulcus. (B) Flat lateral top of the skull, occipital protuberance, low-set ears and low hairline. (C) Clinodactyly of the fifth finger 
microdeletion at Xq22.3-q23 involving the AMMECR1 gene and two cases with CHRDL1 mutation were founded. The clinical features of all reported cases are shown in Table 1.

\section{Discussion}

We found a $671 \mathrm{~KB}$ microdeletion located at Xq23, which involved both the AMMECR 1 and CHRDL1 genes. Although Xq22.3-q23 microdeletion has been reported, to our knowledge, this is the first case only with Xq23 microdeletion. Literature describing disorders associated with AMMECR 1 is also rare. The description and name of the gene (Alport syndrome, mental retardation, midface hypoplasia and elliptocytosis chromosomal region gene 1) is derived from its cytogenic location within Xq22.3-q23 and its previous association with a very peculiar contiguous gene deletion syndrome, originally named AMME (Alport syndrome, mental retardation, midface hypoplasia and eliptocytosis). A deletion including COL4A5 (Xq22.3) extending proximally to include AMMECR 1 which was first named AMME (4). However, the biological function of $A M M E C R 1$ remains unclear. It was reported to code a protein that has a putative nuclear localization signal and may, therefore, encode a factor that regulates transcription (5). Besides, a $250 \mathrm{~KB}$ deletion on Xq23 that involved the CHRDL1 gene and segregated with the affected phenotype in the affected individual's family, these authors described an inherited congenital disorder which was named MGC1. This gene encodes ventroptin, a bone morphogenic protein antagonist, with a proposed role in specification of topographic retinotectal projections and CHRDL1 is differentially expressed in the human fetal brain, with high expression in cerebellum and neocortex (6).

Similar to two previously reported cases with the same mutation at Xq22.3-q23 of the AMMECR 1 gene by Andreoletti et al. (1) the current case presented with microsomia, right kidney dysplasia, mild motor retardation, spoke early but with a stutter, had midface hypoplasia including flat facial profile, mildly epicanthic folds, downslanting palpebral fissures, flat nasal bridge, bulbous nose, and a short neck. This implies that in patients with growth retardation, midface hypoplasia, kidney dysplasia, motor retardation, and language disorder, as well as alteration of bones and heart, Xq23 microdeletion should be considered.

Comparing the phenotype of the current patient with previous cases, we noted that the current case did not show mental retardation or hearing loss and showed a language disorder, which was noted in patients reported by Jonsson et al. (4). However, the current case also presented with bilateral clinodactyly of the fifth finger, cardiac and skeletal abnormalities but did not have elliptocytosis or hearing loss, which was noted in the two cases reported by Andreoletti et al. (1). These authors detailed varying degrees of hearing impairment but without skeletal abnormalities. However, the older brother of these two cases showed scattered elliptocytes and anisocytosis. It was remarkable that the current case presented with short stature, skeletal and cardiac abnormalities, similar to the five cases reported by Moysés-Oliveira et al. (2) but the previous reported patients also had hearing loss while the current case did not. This

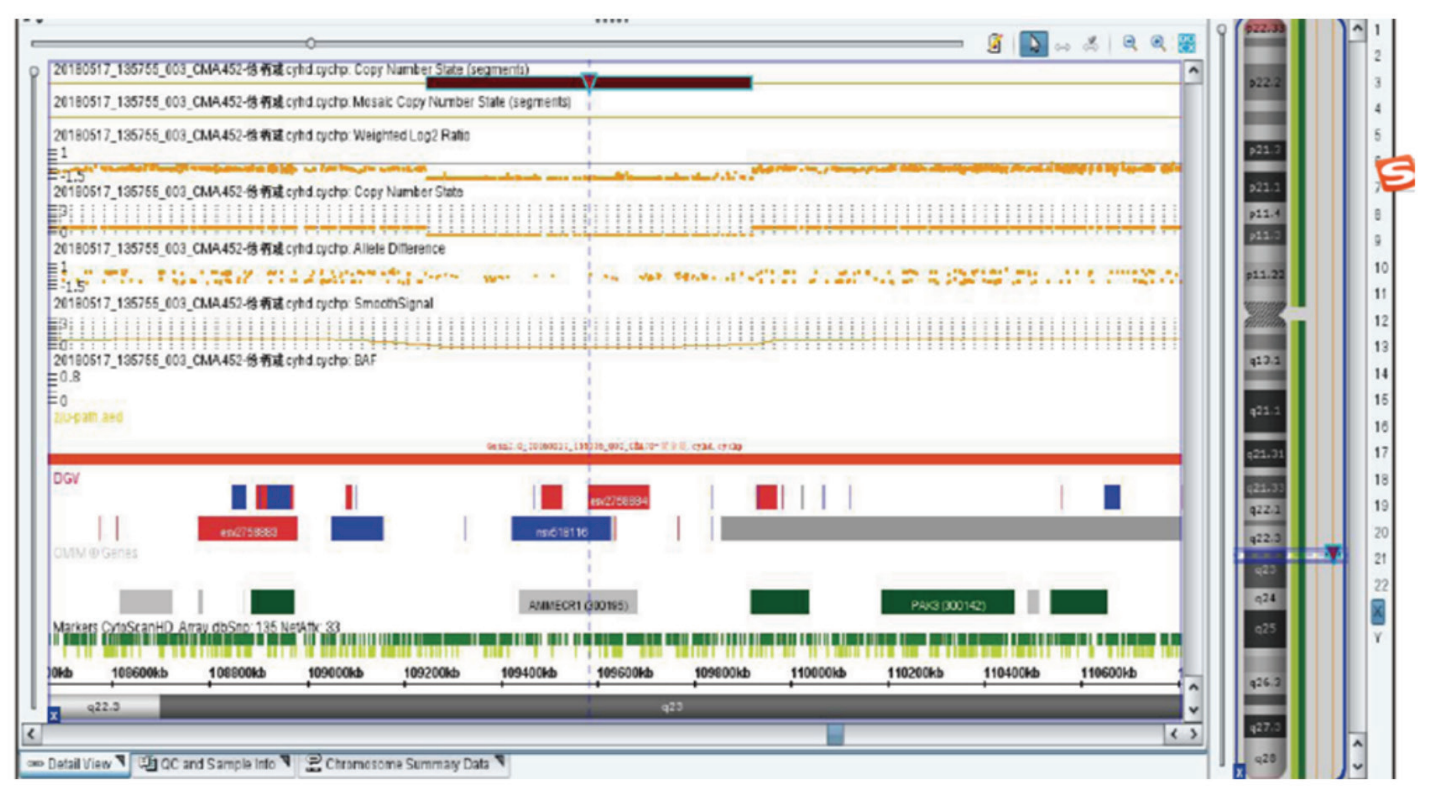

Figure 2. Chromosomal microarray analysis found one $671 \mathrm{~KB}$ microdeletion located at Xq23 that covered the AMMECR1 and CHRDL1 genes 


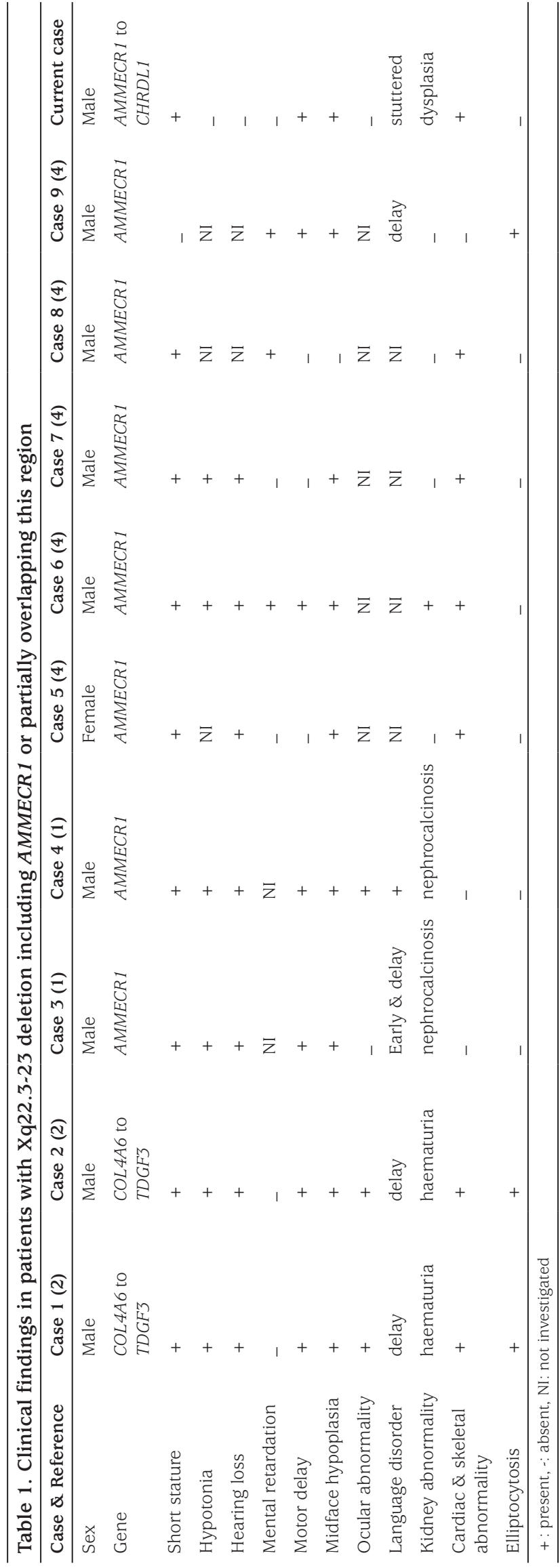

finding on our patient suggested the microdeletion of Xq23 including AMMECR 1 may not be directly related to mental retardation and hearing loss, but it has a direct impact on growth and development, leading to facial malformation and bone and heart alterations. Besides, the phenotype of kidney abnormalities may be related to AMMECR1, but the manifestations of renal pathology are different. Moreover, the current case did not present with megalocornea or other eye lesions that have been associated with mutation of CHRDL1 gene (7). It was notable that the report of Davidson et al. (8) included global developmental delay, midface hypoplasia, walking at the age of 20 months and a need for speech therapy in patients with mutation of CHRDL1. Our patient did not have intellectual disability at assessment while that of Davidson et al. (8) had moderate intellectual disability at the age of 10 years. Of course, the current patient may develop mental retardation later. Thus, given the clinical findings in the current case, microdeletion of Xq23 involving CHRDL1 does not necessarily lead to X-linked megalocornea and intellectual disability. However, mutation of CHRDL1 may lead to the above phenotypes. Besides, comparing the reports of Webb et al. (6) and Davidson et al. (8) with the current patient, we can hypothesize that megalocorneamental retardation (MMR syndrome), in some cases, may be di- or multi-genic, because the current patient did not show any ocular abnormality and mental retardation. The clinical features of Xq22 to Xq23 microdeletion appear to be varied, although development disability, facial malformation, renal abnormality, cardiac and bone alteration are prevalent among individuals with deletions across the Xq22.3-q23 region.

\section{Conclusion}

In summary, Xq23 microdeletion is a rare condition. In patients with growth retardation, midface hypoplasia, kidney dysplasia, motor retardation, and language disorder, as well as alteration of bones and cardiac morphology/ function, Xq23 microdeletion should be considered. A detailed family history, careful physical exam to identify distinctive clinical features and improved genetic diagnosis may directly benefit the patient by allowing management and counseling specific for the disorder (9).

\section{Acknowledgement}

We thank the patient and his parent to allow us using the data.

\section{Ethics}

Informed Consent: Consent form was filled out by all participants. 
Peer-review: Externally peer-reviewed.

\section{Authorship Contributions}

Surgical and Medical Practices: Lu Qin, Concept: Fei-Zhou Zhang, Design: Lu Qin, Data Collection or Processing: JianHai Lv, Analysis or Interpretation: Fei-Zhou Zhang, Literature Search: Lu Qin, Lan-Fang Tang, Writing: Lu Qin.

Financial Disclosure: This work is supported by the National Natural Science Foundation of China (81170016 \& 81470214) and Zhejiang Provincial Program for the Cultivation of High-Level Innovative Health Talents (2016).

\section{References}

1. Andreoletti G, Seaby EG, Dewing JM, O'Kelly I, Lachlan K, Gilbert RD, Ennis S. AMMECR1: a single point mutation causes developmental delay, midface hypoplasia and elliptocytosis. J Med Genet 2017;54:269277. Epub 2016 Nov 3

2. Moysés-Oliveira M, Giannuzzi G, Fish RJ, Rosenfeld JA, Petit F, Soares MF, Kulikowski LD, Di-Battista A, Zamariolli M, Xia F, Liehr T, Kosyakova N, Carvalheira G, Parker M, Seaby EG, Ennis S, Gilbert RD, Hagelstrom RT, Cremona ML, Li WL, Malhotra A, Chandrasekhar A, Perry DL, Taft RJ, McCarrier J, Basel DG, Andrieux J, Stumpp T, Antunes F, Pereira GJ, Neerman-Arbez M, Meloni VA, Drummond-Borg M, Melaragno MI, Reymond A. Inactivation of AMMECR 1 is associated with growth, bone, and heart alterations. Hum Mutat 2018;39:281-291. Epub 2017 Dec 14

3. Mangialavori D, Colao E, Carnevali A, Bruzzichessi D, Grillone T, Perrotti N, Iuliano R, Scorcia V. Novel Mutation in the CHRDL1 Gene Detected in Patients With Megalocornea. Cornea 2015;34:976-979.
4. Jonsson JJ, Renieri A, Gallagher PG, Kashtan CE, Cherniske EM, Bruttini M, Piccini M, Vitelli F, Ballabio A, Pober BR. Alport syndrome, mental retardation, midface hypoplasia, and elliptocytosis: a new $\mathrm{X}$ linked contiguous gene deletion syndrome? J Med Genet 1998;35:273-278.

5. Vitelli F, Piccini M, Caroli F, Franco B, Malandrini A, Pober B, Jonsson $\mathrm{J}$, Sorrentino $\mathrm{V}$, Renieri A. Identification and characterization of a highly conserved protein absent in the Alport syndrome (A), mental retardation $(M)$, midface hypoplasia $(M)$, and elliptocytosis (E) contiguous gene deletion syndrome (AMME). Genomics 1999;55:335340 .

6. Webb TR, Matarin M, Gardner JC, Kelberman D, Hassan H, Ang W, Michaelides M, Ruddle JB, Pennell CE, Yazar S, Khor CC, Aung T, Yogarajah M, Robson AG, Holder GE, Cheetham ME, Traboulsi EI, Moore AT, Sowden JC, Sisodiya SM, Mackey DA, Tuft SJ, Hardcastle AJ. $\mathrm{X}$-linked megalocornea caused by mutations in CHRDL1 identifies an essential role for ventroptin in anterior segment development. Am J Hum Genet 2012;90:247-259. Epub 2012 Jan 26

7. Han J, Young JW, Frausto RF, Isenberg SJ, Aldave AJ. X-linked Megalocornea Associated with the Novel CHRDL1 Gene Mutation p.(Pro56Leu*8). Ophthalmic Genet 2015;36:145-148. Epub 2013 Sep 27

8. Davidson AE, Cheong SS, Hysi PG, Venturini C, Plagnol V, Ruddle JB, Ali H, Carnt N, Gardner JC, Hassan H, Gade E, Kearns L, Jelsig AM, Restori M, Webb TR, Laws D, Cosgrove M, Hertz JM, Russell-Eggitt I, Pilz DT, Hammond CJ, Tuft SJ, Hardcastle AJ. Association of CHRDL1 mutations and variants with X-linked megalocornea, Neuhäuser syndrome and central corneal thickness. PLoS One 2014;9:e104163.

9. Jee YH, Baron J, Nilsson O. New developments in the genetic diagnosis of short stature. Curr Opin Pediatr 2018;30:541-547. 